\title{
Doença de Wilson em crianças e adolescentes: diagnóstico e tratamento
}

\author{
Wilson's disease in children and adolescents: diagnosis and treatment
}

\begin{abstract}
Stephania de Andrade Sócio ${ }^{1}$, Alexandre Rodrigues Ferreira², Eleonora Druve T. Fagundes², Mariza Leitão V. Roquete ${ }^{2}$, Júlio Rocha Pimenta ${ }^{3}$, Lilian de Faria Campos ${ }^{4}$, Francisco José Penna ${ }^{5}$
\end{abstract}

\section{RESUMO}

Objetivo: Descrever as formas de apresentação, as alterações laboratoriais ao diagnóstico e o tratamento de crianças e adolescentes com doença de Wilson.

Métodos: Estudo descritivo e retrospectivo de 17 crianças e adolescentes com doença de Wilson atendidos no Ambulatório de Hepatologia Pediátrica do Hospital das Clínicas da Universidade Federal de Minas Gerais no período de 1985 a 2008. Os dados foram coletados dos prontuários e durante as consultas ambulatoriais.

Resultados: A idade ao diagnóstico variou de 2,8 a 15,1 anos, com média de 8,8 $\pm 0,9$ anos. A forma de apresentação predominante foi hepática ( $53 \%$ ), seguida por assintomáticos provenientes de triagem familiar. $\mathrm{O}$ anel de Kayser-Fleischer foi encontrado em $41 \%$ dos pacientes. A ceruloplasmina encontrava-se alterada em 15/17 pacientes e o cobre urinário variou de 24 a $1000 \mathrm{mcg} / 24 \mathrm{~h}$ (mediana: $184 \mathrm{mcg} / 24 \mathrm{~h}$ ). O tratamento instituído foi a D-penicilamina. Observaramse efeitos colaterais em cinco crianças, sem necessidade de interrupção ou troca da medicação. As respostas clínica e laboratorial, com níveis normais de aminotransferases, foram evidenciadas em 14 pacientes após mediana de 10,7 meses de tratamento. Três crianças morreram (uma por hepatite fulminante e duas com complicações da insuficiência hepática grave), apesar do tratamento.

Conclusões: A doença de Wilson é rara na faixa etária pediátrica. A forma de apresentação predominante é a hepática. Seu diagnóstico se baseia principalmente em dosagem de ceruloplasmina baixa, cobre livre e cobre em urina de 24 horas elevados, mas exige alto grau de suspeição. Apresenta boa resposta e tolerância ao tratamento medicamentoso.

Palavras-chave: doença de Wilson; degeneração hepatolenticular; criança; insuficiência hepática.

\section{ABSTRACT}

Objective: To describe clinical symptoms, laboratory findings at diagnosis and treatment of children and adolescents with Wilson's disease.

Methods: This is a descriptive and retrospective study of a series of 17 children and adolescents with Wilson's disease, assited at the Pediatric Hepatology Ambulatory of the Hospital das Clínicas of Universidade Federal de Minas Gerais, Brazil, from 1985 to 2008. Data were collected by revision of medical charts and during clinical follow-up.

Results: Patients were 2.8 to 15.1 years old, with a mean age of $8.8 \pm 0.9$ years. The disease main presentation was hepatic $(53 \%)$, followed by the asymptomatic form, diagnosed by family screening. The Kayser-Fleischer ring was observed in $41 \%$ of the patients. The ceruloplasmin was altered in 15 out of 17 patients, and the urinary copper varied from 24 to $1000 \mathrm{mcg} / 24 \mathrm{~h}$ (median: $184 \mathrm{mcg} / 24 \mathrm{~h}$ ). The treatment was

Endereço para correspondência:

Eleonora Druve T. Fagundes

Rua Tenente Anastácio de Moura, 740/801 - Santa Efigênia

CEP 30240-390 - Belo Horizonte/MG

E-mail: eleonoradruve@uol.com.br

Conflito de interesses: nada a declarar

Recebido em: 27/5/09

Aprovado em: 31/8/09 
stablished with D-penicillamine in all cases. Slight side effects were observed in five children, with no need to interrupt or change medication. Clinical and laboratory responses to treatment, with normalization of aminotransferases levels, were shown in 14 patients after a median of 10.7 months. Although treated, three patients died (one due to fulminant hepatitis and two due to severe hepatic failure).

Conclusions: Wilson's disease is rare in the pediatric group. In children, the main presentation is the liver disease. The diagnosis can be established by reduced ceruloplasmin levels and elevated copper excretion in the 24-hour urine, but it demands high suspicion level. There are good tolerance and response to medical treatment.

Key-words: Wilson's disease; hepatolenticular degeneration; child; hepatic insufficiency.

\section{Introdução}

A doença de Wilson é uma afecção autossômica recessiva, cuja prevalência estimada é de 1:40.000 pessoas e decorre da mutação do gene ATP 7B, localizado no cromossomo 13. Essa mutação leva à redução na excreção de cobre pelas vias biliares e à sua incorporação à ceruloplasmina, uma glicoproteína que transporta o metal pelo organismo ${ }^{(1)}$. Como consequência, o cobre se acumula em diversos tecidos, como fígado, sistema nervoso central, córneas e rins, gerando lesões hepatocelulares cirrotizantes, demência, distúrbios neuropsiquiátricos, alterações de função renal e cardíaca. A tríade clássica de apresentação é composta pela doença hepática, neurológica e oftalmológica. As manifestações hepáticas predominam na faixa pediátrica. Alterações neurológicas correspondem a 10 a $25 \%$ dos $\operatorname{casos}^{(1)}$, mas são especialmente identificadas em adultos. A impregnação do metal na córnea, o anel de KayserFleischer (KF), é a alteração oftalmológica mais frequente, podendo estar ausente nas crianças e ter relação com o quadro neuropsiquiátrico ${ }^{(1-3)}$. São descritas alterações mais raras como renais (proteinúria, hematúria, litíase), ósteo-articulares (osteopenia, artralgias, artrite), hematológicas (hemólise), cardíacas (arritmias, hipertrofia ventricular, morte súbita) e neoplásicas (adenocarcinoma, hepatoblastoma) ${ }^{(1)}$.

A doença de Wilson é uma hepatopatia rara, mas seu diagnóstico apresenta grande impacto, uma vez que há tratamento específico disponível, comprovadamente eficaz e sem o qual é invariavelmente fatal. O tratamento precoce evita graves complicações. O diagnóstico pode ser difícil, pois não há um único exame com sensibilidade adequada e as manifestações podem não ser típicas, especialmente entre as crianças. Depende de alto grau de suspeição clínica diante de um paciente com doença hepática e/ou neuropsiquiátrica ${ }^{(4)}$. O diagnóstico baseia-se em alterações laboratoriais como ceruloplasmina baixa, cobre urinário de 24 horas, cobre livre e dosagem de cobre no tecido hepático elevados. Ao exame oftalmológico, a presença de anel de KF reafirma o diagnóstico ${ }^{(1)}$.

O tratamento baseia-se no uso de drogas quelantes do cobre. A D-penicilamina é a droga de escolha, apesar do risco de piora neurológica em até $50 \%$ dos pacientes e dos diversos efeitos colaterais associados ao seu uso ${ }^{(1,4)}$. A trientina e o tetratiomolibdato são drogas alternativas, sendo a última escolhida para indivíduos com sintomas neurológicos ${ }^{(1)}$. O zinco tem indicação em assintomáticos ou em terapia de manutenção ${ }^{(5)}$.

Existem poucas publicações com casuística exclusivamente pediátrica ${ }^{(4,6-10)}$. Este estudo teve como objetivo descrever as formas de apresentação clínica, alterações laboratoriais e resposta ao tratamento em crianças e adolescentes com doença de Wilson.

\section{Método}

Trata-se de estudo retrospectivo e descritivo de série de casos de crianças e adolescentes com diagnóstico de doença de Wilson atendidos no Ambulatório de Hepatologia Pediátrica do Hospital das Clínicas da Universidade Federal de Minas Gerais (UFMG), entre 1985 e 2008.

Foram incluídos 17 pacientes com idade inferior a 18 anos. Os dados foram coletados de revisão de prontuários arquivados no Serviço de Arquivos Médicos do Hospital das Clínicas da UFMG e ao longo das consultas nos retornos ambulatoriais.

As variáveis estudadas foram: idade ao diagnóstico, gênero, formas de apresentação clínica, exames laboratoriais ao diagnóstico, presença de anel de KF, ultrassonografia abdominal, endoscopia digestiva alta (EDA), biópsias hepáticas, tempo para normalização dos níveis de aminotransferases após início do tratamento, tratamento instituído e seus efeitos colaterais.

As manifestações clínicas foram definidas como:

- Forma assintomática: caracterizada pela ausência de sinais e sintomas de doença hepática, neurológica ou oftalmológica, mas com alterações laboratoriais compatíveis com a doença de Wilson.

- Forma hepática aguda, crônica e fulminante: 
a) Hepatite aguda: semelhante a uma hepatite viral com icterícia, colúria, hepatomegalia e elevação de aminotransferases.

b) Hepatite crônica: sinais de hipertensão portal, hepatomegalia, esplenomegalia, elevação de enzimas hepáticas, com ou sem icterícia.

c) Insuficiência hepática fulminante: manifestações clínicas de hepatite aguda e aparecimento de encefalopatia até oito semanas após o surgimento de manifestações clínicas da doença hepática.

- Forma neurológica: caracterizada por alterações neuropsiquiátricas, como mudanças de comportamento, psicoses e distúrbios da fala, entre outras.

O diagnóstico de doença de Wilson foi baseado na presença de pelo menos dois dos seguintes critérios: (1) história familiar positiva; (2) presença de anel de KF; (3) ceruloplasmina reduzida (<20mg/dL); (4) cobre livre maior de $25 \mu \mathrm{g} / \mathrm{dL}$ [calculado a partir da fórmula: cobre livre $=$ cobre sérico em $\mathrm{mcg} / \mathrm{dL})-(3$ x ceruloplasmina em mg/dL)]; (5) cobre urinário de 24 horas acima de $100 \mu \mathrm{g} / 24 \mathrm{~h}$. A dosagem de cobre no tecido hepático seco não é realizada no Serviço.

O diagnóstico também foi firmado nos pacientes com doença hepática crônica que apresentavam pelo menos um teste do metabolismo do cobre alterado e boa resposta ao tratamento com quelante, uma vez excluídas outras hepatopatias crônicas. Hepatite autoimune, hepatite crônica pelos

Tabela 1 - Critérios de Nazer et a/(11) de classificação prognóstica de acordo com a função hepática

\begin{tabular}{lccc}
\hline Pontos & Bilirrubina $(\mathbf{m g} / \mathbf{d L})$ & AST $(\mathbf{U I} / \mathbf{L})$ & RNI \\
\hline 0 & $<5,8$ & $<100$ & $<1,3$ \\
1 & $5,9-8,8$ & $100-150$ & $1,3-1,6$ \\
2 & $8,9-11,7$ & $151-200$ & $1,6-1,9$ \\
3 & $11,8-17,5$ & $201-300$ & $1,9-2,4$ \\
4 & $>17,5$ & $>300$ & $>2,4$ \\
\hline
\end{tabular}

Escore $>7$ sugere alto risco de mortalidade se não for submetido a transplante hepático. AST: dosagem sérica de aspartato aminotransferase; RNI: razão normalizada internacional do tempo de protrombina. vírus $\mathrm{B}$ e $\mathrm{C}$ e deficiência de $\alpha 1$-antitripsina foram excluídas em todos os pacientes.

O exame oftalmológico foi realizado em todos os pacientes com lâmpada de fenda à procura de anel de KF e catarata em girassol. Exames de imagem, como ultrassonografia abdominal e EDA, também foram realizados, de acordo com a indicação clínica - a EDA foi indicada na presença de sinais de hipertensão porta. Os exames laboratoriais solicitados ao diagnóstico foram: dosagem sérica de aspartato aminotransferase (AST) e alanina aminotransferase (ALT), bilirrubina total e frações e albumina. Os valores das aminotransferases foram considerados alterados quando acima do maior valor de referência (MVR).

O tratamento foi instituído com D-penicilamina para todos os pacientes, com doses iniciais de $10 \mathrm{mg} / \mathrm{kg} / \mathrm{dia}$ (ou $250 \mathrm{mg} / \mathrm{dia}$ ), aumentadas para $20 \mathrm{mg} / \mathrm{kg} /$ dia após 30 dias do seu início. A dose máxima recomendada é de 1000 a $1500 \mathrm{mg} / \mathrm{dia}$, dividida em três tomadas. O objetivo é manter o cobre urinário de 24 horas entre 200 e $500 \mathrm{mcg} / 24 \mathrm{~h}$ e cobre livre abaixo de $10 \mathrm{mcg} / \mathrm{dL}$. A piridoxina foi usada simultaneamente na dose de $25 \mathrm{mg} /$ dia.

O acompanhamento ambulatorial foi mensal nos seis primeiros meses; bimestral entre seis a 12 meses após o início do tratamento; e trimestral a seguir. A monitorização laboratorial do tratamento foi realizada com dosagem do cobre urinário de 24 horas, cobre livre, avaliação da função hepática, hemograma, contagem de plaquetas e qualitativo de urina para controle dos efeitos adversos. A triagem familiar foi realizada em todos os pais e irmãos dos pacientes com diagnóstico de doença de Wilson.

Para avaliar a gravidade e predizer a evolução dos pacientes com doença de Wilson, foram calculados os escores de Dhawan $e t a l^{(8)}$ e Nazer $e t a l^{(1)}$, conforme as Tabelas 1 e 2.

A análise dos dados foi efetuada com os recursos estatísticos do software Epi-Info 6.04. As variáveis foram expressas em média, desvio-padrão, mediana e intervalo interquartil 25-75\% (IQ25-75\%). O estudo foi aprovado pelo Comitê de Ética em Pesquisa da UFMG.

Tabela 2 - Índice preditor de mortalidade para doença de Wilson proposto por Dhawan et al.(8)

\begin{tabular}{lccccc}
\hline Pontos & Bilirrubina $(\mathbf{m g} / \mathbf{d L})$ & AST $(\mathbf{U I} / \mathbf{L})$ & RNI & Leucócitos (109/L) & Albumina (g/L) \\
\hline 0 & $<5,8$ & $<100$ & $<1,29$ & $0-6,7$ & $>45$ \\
1 & $5,9-8,8$ & $100-150$ & $1,3-1,6$ & $6,8-8,3$ & $34-44$ \\
2 & $8,9-11,7$ & $151-300$ & $1,7-1,9$ & $8,4-10,3$ & $25-33$ \\
3 & $11,8-17,5$ & $301-400$ & $2,0-2,4$ & $10,4-15,3$ & $21-24$ \\
4 & $>17,5$ & $>401$ & $>2,5$ & $>15,4$ & $<20$ \\
\hline
\end{tabular}

Escore $\geq 10$ preditor de mortalidade se não submetido a transplante hepático. AST: dosagem sérica de aspartato aminotransferase; RNI: razão normalizada internacional do tempo de protrombina. 


\section{Resultados}

Foram avaliadas 17 crianças e adolescentes, sendo dez (59\%) do gênero feminino, com idade ao diagnóstico variando de 2,8 a 15,1 anos e média de $8,8 \pm 0,9$ anos.

A forma de apresentação foi predominantemente hepática (11/17 pacientes, 65\%), distribuídos em seis casos de hepatite aguda (um com a forma fulminante) e cinco de hepatite crônica. Dois pacientes (12\%) apresentaram também quadro de glomerulonefrite. Seis pacientes (35\%) foram identificados a partir de triagem familiar, estando todos assintomáticos, mas com alterações de aminotransferases.

Os principais achados laboratoriais ao diagnóstico foram:

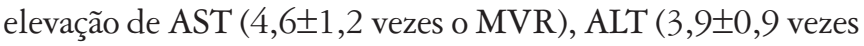
o MVR) e bilirrubina total $(5,3 \pm 3,4 \mathrm{mg} / \mathrm{dL})$. A dosagem de albumina variou entre 2,3 e 5,0, com média de $3,8 \pm 0,7 \mathrm{~g} / \mathrm{dL}$. O cobre urinário variou de 24 a $1000 \mathrm{mcg} / 24 \mathrm{~h}$, com mediana de $184 \mathrm{mcg} / 24 \mathrm{~h}(\mathrm{p} 25 \%=106$ e p $75 \%=497)$ e o cobre livre variou de 1,8 a $119 \mu \mathrm{g} / \mathrm{dL}$, com mediana de $27 \mathrm{mcg} / \mathrm{dL}$ (p25\%=20 e $\mathrm{p} 75 \%=4,1)$. A ceruloplasmina variou de 1 a $47 \mathrm{mg} / \mathrm{dL}$, com mediana de $4 \mathrm{mg} / \mathrm{dL}$ (p25\%=3 e p $75 \%=8$ ).

Todos os pacientes foram submetidos a exame oftalmológico e o anel de KF foi detectado em sete (41\%) - todos com doença hepática grave ou crônica e com idades que variavam entre sete a 12 anos (média: 10,6 anos). As características clínicas e laboratoriais dos pacientes estão na Tabela 3.

Durante o tempo de seguimento, 14 pacientes foram submetidos à ultrassonografia abdominal e 64\% apresentavam alterações como hepatoesplenomegalia e sinais de cirrose. Sete foram submetidos à EDA pois demonstravam sinais de hipertensão porta; $43 \%$ exibiam varizes esofágicas.

Apenas quatro pacientes foram submetidos à biópsia hepática. Uma criança de cinco anos apresentava alterações discretas e inespecíficas. Outra, de nove anos, mostrava necrose erosiva e cirrose. As demais foram realizadas em duas crianças de dez anos, uma com esteatose hepática e fibrose discreta e outra com hepatite crônica inflamatória com infiltrado linfocitário e fibrose moderada.

O tratamento medicamentoso foi administrado em 16 dos 17 pacientes (houve um óbito por hepatite fulminante) com D-penicilamina, em doses que variaram de 250 a $750 \mathrm{mg}$ ao dia. Desses 16 pacientes, cinco $(31 \%)$ apresentaram efeitos colaterais, como cefaleia (1/16), plaquetopenia (1/16), proteinúria (1/16), náuseas e vômitos (2/16) e dor em membros (1/16). Esses efeitos, entretanto, foram transitórios, não sendo necessária a suspensão ou a troca do medicamento.

A idade do início do tratamento variou de 2,8 a 15 anos,

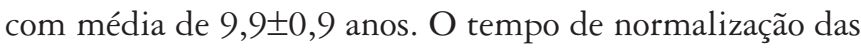

Tabela 3 - Características clínicas e laboratoriais dos 17 pacientes com doença de Wilson

\begin{tabular}{|c|c|c|c|c|c|c|c|}
\hline Paciente & $\begin{array}{l}\text { Idade } \\
\text { (anos) }\end{array}$ & $\begin{array}{c}\text { Gênero } \\
\text { (M/F) }\end{array}$ & $\begin{array}{l}\text { Ceruloplasmina } \\
\text { (mg/dL) }\end{array}$ & $\begin{array}{l}\text { Cobre urinário } \\
\text { (mcg/24h) }\end{array}$ & $\begin{array}{c}\text { Cobre livre } \\
\text { (mcg/dL) }\end{array}$ & $\begin{array}{c}\text { Anel } \\
\text { de KF }\end{array}$ & $\begin{array}{l}\text { Apresentação } \\
\text { clínica }\end{array}$ \\
\hline 1 & 8 & $\mathrm{M}$ & 2,0 & 772,5 & 118,7 & $\mathrm{~N}$ & $\begin{array}{l}\text { Hepatite aguda, } \\
\text { GNDA }\end{array}$ \\
\hline 2 & 2 & $\mathrm{~F}$ & 4,0 & 15,5 & 1,8 & $\mathrm{~N}$ & Assintomático, $\mathrm{HF}$ \\
\hline $3^{*}$ & 10 & $\mathrm{~F}$ & 3,0 & 164,0 & 12,3 & $S$ & Assintomático \\
\hline 4 & 14 & $\mathrm{~F}$ & 2,0 & 240,0 & 26,7 & $\mathrm{~N}$ & Assintomático \\
\hline 5 & 7 & $\mathrm{~F}$ & 1,0 & 188,0 & 24,9 & $\mathrm{~N}$ & Assintomático \\
\hline 6 & 13 & $M$ & 3,0 & 884,4 & 78,6 & $\mathrm{~N}$ & Hepatite crônica \\
\hline $7^{*}$ & 9 & $\mathrm{M}$ & 4,8 & 98,3 & 22,8 & $\mathrm{~N}$ & Hepatite crônica \\
\hline 8 & 10 & $\mathrm{~F}$ & 5,6 & 183,6 & 30,8 & $S$ & $\begin{array}{l}\text { Hepatite aguda, } \\
\text { GNDA }\end{array}$ \\
\hline $9^{*}$ & 10 & $F$ & 7,0 & 1000,0 & 19,9 & $\mathrm{~N}$ & Hepatite crônica \\
\hline 10 & 12 & $\mathrm{~F}$ & 10,0 & 1000,0 & 80,5 & $S$ & $\begin{array}{l}\text { Hepatite aguda } \\
\text { fulminante }\end{array}$ \\
\hline 11 & 11 & $\mathrm{M}$ & 8,0 & 645,4 & 23,7 & $S$ & Hepatite aguda \\
\hline $12^{*}$ & 5 & $\mathrm{~F}$ & 4,4 & 24,4 & 41,1 & $\mathrm{~N}$ & Assintomático, $\mathrm{HF}$ \\
\hline 13 & 7 & $\mathrm{M}$ & 7,3 & 453,0 & 27,8 & $S$ & Hepatite aguda \\
\hline 14 & 11 & $\mathrm{~F}$ & 47,0 & 55,0 & 32,1 & $S$ & Hepatite crônica \\
\hline 15 & 14 & $M$ & 38,0 & 64,7 & 44,7 & $\mathrm{~N}$ & Hepatite aguda \\
\hline 16 & 11 & $\mathrm{M}$ & 3,0 & 497,4 & 20,4 & $S$ & Hepatite crônica \\
\hline 17 & 3 & $\mathrm{~F}$ & 8,89 & 106,0 & 10,0 & $\mathrm{~N}$ & Assintomático, HF \\
\hline
\end{tabular}

F: feminino; M: masculino; S: sim; N: não; GNDA: glomerulonefrite difusa aguda; HF: história familiar. *Pacientes submetidos à biópsia hepática percutânea. 
aminotransferases variou de um a 24 meses, com mediana de 11 meses (p25\%=3 e p $75 \%=12)$ e refere-se a 14 dos 16 pacientes que iniciaram tratamento. Dois pacientes não chegaram a normalizar os exames e morreram em razão de complicações da hepatopatia crônica grave. A taxa total de óbito foi de $18 \%$ (3/17).

A Tabela 4 evidencia as pontuações de gravidade de acordo com os critérios de Nazer e Dhawan para os pacientes da casuística. Entre os três pacientes que faleceram, apenas um apresentava escore de Nazer maior que 7; dois apresentavam escore de Dhawan igual a 10. Um paciente com escore 10 de Dhawan apresentou melhora clínica após seis meses de tratamento com D-penicilamina.

\section{Discussão}

A doença de Wilson é uma causa rara de hepatopatia na criança. Existem poucos trabalhos que descrevem casuísticas exclusivamente pediátricas e os que o fazem geralmente apresentam pequeno número de pacientes - como o de SánchezAlbisua $e t a l^{(4)}$, com 26 crianças, e o de Yüce et $a l^{(7)}, \operatorname{com} 33$ crianças. A maior casuística é a de Dhawan et al ${ }^{(8)}$, do King's College Hospital, em Londres, com 74 crianças em 37 anos.

A idade mais comum ao diagnóstico é a segunda década de vida, sendo raro o achado em menores de cinco anos e maiores de 40 anos $^{(12-14)}$. No entanto, a triagem da doença entre os familiares pode diminuir essa média, uma vez que identifica pacientes assintomáticos mais jovens. $\mathrm{Na}$ presente casuística, o diagnóstico mais precoce, realizado a partir de triagem familiar, ocorreu em uma criança de dois anos de idade e assintomática, mas com alterações de aminotransferases. A média de idade ao diagnóstico neste trabalho foi de 8,8 anos, semelhante à relatada em outros estudos pediátricos ${ }^{(4,7)}$. Na casuística de Sánchez-Albisua et $a l^{(4)}$, a idade ao diagnóstico foi de 9,8 $\pm 3,4$ anos e, no trabalho de Yüce et al ${ }^{(7)}, 10,1 \pm 2,5$ anos.

No que se refere às formas de apresentação, a hepática é a mais prevalente nessa faixa etária, como observado neste trabalho $(65 \%)$. Yüce et al ${ }^{(7)}$ observaram seis casos de hepatite fulminante em 33 crianças com doença de Wilson. Os autores enfatizam a importância de investigar a doença diante dos quadros fulminantes, que parecem ser mais frequentes na segunda década de vida ${ }^{(4,7)}$. No presente estudo, houve um caso de hepatite fulminante em uma menina de 12 anos, que faleceu no período pós-transplante imediato.

Encontrou-se o anel de KF em $41 \%$ dos pacientes, com média de idade de 10,4 anos. A média de idade dos pacientes que não exibiam o sinal foi menor ( 8,5 anos). $\mathrm{O}$ anel de $\mathrm{KF}$ é menos frequentemente encontrado na faixa pediátrica, pois a impregnação do metal depende principalmente do tempo, com incidência de 5,6 a 63\% em casuísticas pediátricas ${ }^{(4,7)}$. Dessa forma, sua ausência não afasta o diagnóstico e sua pre-

Tabela 4 - Pontuações de Nazer et a/(11) e Dhawan et a/(8), apresentação clínica e evolução dos 17 pacientes

\begin{tabular}{lccll}
\hline Paciente & $\begin{array}{c}\text { Pontuação } \\
\text { Nazer }\end{array}$ & $\begin{array}{c}\text { Pontuação } \\
\text { Dhawan }\end{array}$ & Apresentação & Evolução \\
\hline 1 & 2 & 4 & Hepatite aguda, GNDA & Boa resposta \\
2 & 0 & 0 & Assintomático & Boa resposta \\
3 & 0 & 0 & Assintomático & Boa resposta \\
4 & 1 & 1 & Assintomático & Boa resposta \\
5 & 1 & 4 & Assintomático & Boa resposta \\
6 & 7 & 10 & Hepatite crônica & Nazer 0 Dhawan 2 após 6 \\
7 & 4 & & & meses de tratamento \\
8 & 1 & 4 & Hepatite crônica & Boa resposta \\
9 & 2 & 3 & Hepatite aguda, GNDA & Boa resposta \\
10 & 7 & 10 & Hepatite aguda fulminante & Boa resposta \\
11 & 5 & 10 & Hepatite aguda & Óbito \\
12 & 0 & 0 & Assintomático & Boa resposta \\
13 & 6 & 8 & Hepatite aguda & Perdeu seguimento \\
14 & 8 & 9 & Hepatite crônica & Óbito \\
15 & 6 & 7 & Hepatite aguda & Boa resposta \\
16 & 2 & 4 & Hepatite crônica & Boa resposta \\
17 & 5 & 5 & Assintomático & Boa resposta \\
\hline
\end{tabular}

GNDA: glomerulonefrite difusa aguda. 
sença vem sendo relacionada à apresentação neuropsiquiátrica e à maior gravidade da doença hepática ${ }^{(3)}$. Nesta casuística, todos os pacientes que faleceram apresentavam anel de KF.

A forma neurológica tem como manifestações tremores, disartria, ataxia, rigidez, sintomas psiquiátricos, entre outros $^{(1)}$, sendo observada em taxas de $25^{(15)}$ a $71 \%{ }^{(16)} \mathrm{em}$ adultos. Na infância, tal apresentação é menos comum, descrita em taxas de 4 a $12 \%{ }^{(4,7)}$. Nenhum dos pacientes desta casuísica apresentava alterações neurológicas.

Entre os exames laboratoriais, encontra-se a ceruloplasmina como o mais frequentemente alterado (88\%), seguido pela dosagem de cobre urinário de 24 horas $(71 \%)$. Nas séries pediátricas, a ceruloplasmina tem apresentado sensibilidade de 82 a $88 \%$, enquanto a dosagem de cobre em urina de 24 horas mostra sensibilidade de 81 a $100 \%^{(4,7)}$. Tais achados evidenciam a importância da dosagem de ceruloplasmina, de cobre sérico livre e urinário na tentativa de aumentar a sensibilidade do diagnóstico diante de casos suspeitos. Basear-se em apenas um desses exames pode causar falsos-negativos, atrasando o diagnóstico e comprometendo o prognóstico.

A biópsia hepática, por ser um exame pouco específico, não é essencial para o diagnóstico, exceto pela dosagem de cobre tecidual, que é significativa quando elevada. No entanto, a dosagem do cobre tecidual pode apresentar resultado falsonegativo em crianças, uma vez que depende do tamanho da amostra e do tempo de impregnação do metal, que pode estar irregularmente distribuído ${ }^{(4,7,16)}$.

O tratamento adequado deve ser iniciado precocemente para evitar ou minimizar os efeitos nocivos da impregnação do cobre nos diversos tecidos. A dieta de restrição de alimentos que contêm grande quantidade de cobre é adjuvante no tratamento. O tratamento farmacológico é realizado com drogas quelantes de cobre, sendo a mais utilizada e estudada a D-penicilamina, que, no entanto, apresenta uma série de efeitos colaterais, como hipersensibilidade, supressão medular, desenvolvimento de doenças autoimunes, deterioração neurológica, nefrotoxicidade, polineuropatia, neurite óptica e polimiosite ${ }^{(2,9)}$. A D-penicilamina foi iniciada em 16 dos 17 pacientes avaliados neste trabalho, sendo bem tolerada, sem efeitos colaterais graves ou necessidade de suspensão da droga. Dhawan et al ${ }^{(8)}$ identificaram $3 / 57$ pacientes com supressão medular após uso de D-penicilamina, sendo necessária a sua troca por trientina. Na casuística aqui avaliada, não foi necessário trocar a medicação em nenhum paciente. Todos continuaram em uso de D-penicilamina.

O transplante hepático está indicado nas formas de apresentação fulminante, para pacientes com insuficiência hepática grave que não respondem ao tratamento e em casos de complicações da hipertensão porta ${ }^{(3)}$.

A boa resposta ao tratamento medicamentoso é considerada após a normalização dos exames de função hepática ${ }^{(4,17)}$. Neste estudo, o tempo para resposta clínica apresentou mediana de 10,7 meses após o início do uso de D-penicilamina, sendo similar ao encontrado na literatura ${ }^{(4,9,17)}$. Estes dados ressaltam a necessidade de se aguardar um tempo maior para avaliar a resposta ao tratamento, desde que o paciente apresente função estável.

A fim de predizer a evolução dos pacientes com doença de Wilson, alguns critérios de gravidade vêm sendo estudados e o escore de prognóstico proposto por Nazer et al ${ }^{(11)}$, em 1986, é o mais amplamente citado. Tal critério se baseia em pesquisa que envolve tanto crianças quanto adolescentes e adultos, com o objetivo de diferenciar aqueles que provavelmente responderão de forma insatisfatória ao tratamento com quelante e terão risco de morte mais alto sem o transplante hepático. Em 2005, Dhawan et a ${ }^{(8)}$ revisaram o índice prognóstico de Nazer, com base em uma casuística pediátrica com 74 crianças (média de 11,9 anos), e propuseram acrescentar a contagem de leucócitos e a albumina sérica, além mudar o ponto de corte do escore (de $>7$ para $\geq 10$ ), de forma a elevar a especificidade do teste. Os índices de Nazar et al..$^{(11)} \mathrm{e}$ Dhawan et al ${ }^{(8)}$ podem ser úteis na tomada de decisões, uma vez que alguns estudos demonstram que o escore de ChildPugh não é adequado para indicação de transplante hepático em portadores de doença de Wilson ${ }^{(17)}$. No presente estudo, o escore de Dhawan et $a l^{(8)}$ foi mais sensível para identificar pacientes graves (dois dos três pacientes que faleceram apresentavam escore igual ou maior 10). No entanto, um paciente com escore igual a 10 evoluiu com melhora clínica após tratamento medicamentoso. A casuística reduzida não permite maiores extrapolações, mas a avaliação global do paciente, juntamente às pontuações, é necessária para a correta condução dos casos.

A doença de Wilson é uma enfermidade rara e seu diagnóstico é um desafio para pediatras e hepatologistas, pois pode se apresentar de forma oligossintomática e com exames pouco alterados. O diagnóstico depende da observação de dados clínicos e laboratoriais, que evidenciam o metabolismo anormal de cobre, mas nenhum parâmetro é confiável isoladamente. O diagnóstico laboratorial baseia-se na dosagem sérica de ceruloplasmina $(<20 \mathrm{mg} / \mathrm{dL})$, cobre em urina de 24 horas $(>100 \mathrm{mcg} / \mathrm{dL})$ e cobre livre $(>25 \mathrm{mcg} /$ dL). Entretanto, muitos pacientes pediátricos não apresentam essa tríade. O erro mais frequente é pensar que todos 
os parâmetros devem estar alterados. A doença precisa ser considerada em qualquer indivíduo, em qualquer idade, quando há anormalidades hepáticas ou neurológicas ${ }^{(4)}$.

\section{Referências bibliográficas}

1. Roberts EA, Schilsky ML; Division of Gastroenterology and Nutrition, Hospital for Sick Children, Toronto, Ontario, Canada. A practice guideline on Wilson disease. Hepatology 2003;37:1475-92.

2. Hassan A, Masood F. Wilson's disease: a review. J Pak Med Assoc 2004;54:479-84.

3. Schilscky ML. Diagnosis and treatment of Wilson's disease. Pediatr Transpl 2002;6:15-9.

4. Sánchez-Albisua I, Garde T, Hierro L, Camarena C, Frauca E, de la Vega A et al. A high index of suspicion: the key to an early diagnosis of Wilson's disease in childhood. J Pediatr Gastroenterol Nutr 1999;28:186-90.

5. Walshe JM, Munro NA. Zinc-induced deterioration in Wilson's disease aborted by treatment with penicillamine, dimercaprol, and a novel zero copper diet. Arch Neurol 1995;52:10-1.

6. Tissières P, Chevret L, Debray D, Devictor D. Fulminant Wilson's disease in children: appraisal of a critical diagnosis. Pediatr Crit Care Med 2003;4:338-43.

7. Yüce A, Koçak N, Demir H, Gürakan F, Ozen H, Saltik IN et al. Evaluation of diagnostic parameters of Wilson's disease in childhood. Indian J Gastroenterol 2003;22:4-6.

8. Dhawan A, Taylor RM, Cheeseman P, de Silva P, Katsiyiannakis L, Mieli-Vergani G. Wilson's disease in children: 37-year experience and revised King's score for liver transplantation. Liver Transpl 2005;11:441-8.

9. Arnon R, Calderon JF, Schilsky M, Emre S, Shneider BL. Wilson disease in children: serum aminotransferases and urinary copper on trienthylene
A principal exigência para estabelecer o diagnóstico da doença é pensar na sua possibilidade, mantendo alto grau de suspeição. tetramine dihydrochloride (trientine) treatment. J Pediatr Gastroenterol Nutr 2007;44:596-602.

10. Marcellini M, Di Ciommo V, Callea F, Devito R, Comparcola D, Sartorelli MR et al. Treatment of Wilson's disease with zinc from the time of diagnosis in pediatric patients: a single-hospital, 10-year follow-up study. J Lab Clin Med 2005;145:139-43.

11. Nazer H, Ede RJ, MowatAP, Williams R. Wilson's disease: clinical presentation and use of prognostic index. Gut 1986;27:1377-81.

12. Hui J, Fung EL, Tang NL, Chan MH, To KF, Fok TF. Diagnosing Wilson's disease in a 5-year-old child. J Paediatr Child Health 2002;38: 412-3.

13. Wilson DC, Phillips MJ, Cox DW, Roberts EA. Severe hepatic Wilson's disease in preschool-aged children. J Pediatr 2000;137:719-22.

14. Ala A, Borjigin J, Rochwarger A, Schilsky M. Wilson disease in septuagenarian siblings: Raising the bar for diagnosis. Hepatology 2005;41:668-70.

15. Kumagi T, Horiike N, Michitaka K, Hasebe A, Kawai K, Tokumoto Y et al. Recent clinical features of Wilson's disease with hepatic presentation. J Gastroenterol 2004;39:1165-9.

16. Ferenci P, Caca K, Loudianos G, Mieli-Vergani G, Tanner S, Sternlieb I et al. Diagnosis and phenotypic classification of Wilson disease. Liver Int 2003;23:139-42.

17. Brewer GJ. Tetrathiomolybdate anticopper therapy for Wilson's disease inhibits angiogenesis, fibrosis and inflammation. J Cell Mol Med 2003;7:11-20. 